\title{
Characterization of very wide companion candidates to young stars with planets and disks ${ }^{\star}$
} \author{
R. Gratton ${ }^{2}$, and D. Mesa ${ }^{2}$ \\ ${ }^{1}$ Dipartimento di Fisica, dell'Universitá di Roma Sapienza, P.le A. Moro 5, Roma 00185, Italy \\ e-mail: zahra.majidi@roma1.infn.it \\ 2 INAF-Osservatorio Astronomico di Padova, vicolo dell'Osservatorio 5, 35122 Padova, Italy \\ 3 INAF-Osservatorio Astronomico di Capodimonte, via Moiariello 16, 80131 Napoli, Italy \\ 4 INAF-Osservatorio Astrofisico di Catania, via S. Sofia 78, 95123 Catania, Italy \\ ${ }^{5}$ Université Grenoble Alpes, CNRS, IPAG, 38000 Grenoble, France
}

F. Z. Majidi ${ }^{1,2}$, S. Desidera ${ }^{2}$, J. M. Alcalá ${ }^{3}$, A. Frasca ${ }^{4}$, V. D’Orazi ${ }^{2}$, M. Bonnefoy ${ }^{5}$, R. Claudi $^{2}$,

Received 25 July 2020 / Accepted 23 October 2020

\begin{abstract}
Discovering wide companions of stellar systems allows us to constrain the dynamical environment and age of the latter. We studied four probable wide companions of four different stellar systems. The candidates were selected mainly based on their similar kinematic properties to the central star using Gaia DR2. The central stars are V4046 Sgr, HIP 74865, HIP 65426, and HIP 73145, and their probable wide companions are 2MASS J18152222-3249329, 2MASS J15174874-3028484, 2MASS J13242119-5129503, and 2MASS J14571503-3543505 respectively. V4046 Sgr is a member of $\beta$-Pictoris Moving Group while the rest of the stellar systems are acknowledged as members of the Scorpius-Centaurus association. The selected stellar systems are particularly interesting because all of them are already known to possess a low-mass companion and/or a spatially resolved disk. Identifying wider companions of these stars can improve their eligibility as benchmarks for understanding the formation channels of various triple systems, and can help us to determine the orbits of their possibly undiscovered inner, wider companions in case of higher multiplicity. By analyzing the X-shooter spectra of the wide companion candidates of these stars, we obtained their stellar parameters and determined their ages. We find that 2MASS J15174874-3028484 (0.11 $\left.M_{\odot}, 7.4 \pm 0.5 \mathrm{Myr}\right)$, an already recognized pre-main sequence (PMS) member of ScorpiusCentaurus association, is a highly probable wide companion of HIP 74865. 2MASS J13242119-5129503 $\left(0.04 M_{\odot}, 16 \pm 2.2 \mathrm{Myr}\right)$ is ruled out as a plausible wide companion of HIP 65426, but confirmed to be a new sub-stellar member of the Scorpius-Centaurus association. 2MASS J14571503-3543505 $\left(0.02 M_{\odot}, 17.75 \pm 4.15 \mathrm{Myr}\right)$ is a probable sub-stellar member of the same association, but we cannot confirm whether or not it is gravitationally bound to HIP 73145. 2MASS J18152222-3249329 (0.3 $M_{\odot}$, older than 150 Myr) is determined to be a mildly active main sequence star, much older than members of $\beta$-Pictoris Moving Group, and unbound to V4046 Sgr despite their similar kinematic features. PMS wide companions such as 2MASS J15174874-3028484 might have formed through cascade fragmentation of their natal molecular core, hinting at high multiplicity in shorter separations which can be confirmed with future observations.
\end{abstract}

Key words. stars: pre-main sequence - stars: low-mass - protoplanetary disks - ISM: individual objects: HIP 65426 ISM: individual objects: HIP 74865

\section{Introduction}

The characterization of the wide companions of stellar systems is of great interest for various reasons in astronomy and astrophysics. While the discovery of our closest neighbor, Proxima, dates back to more than one century ago (Innes 1915), soon followed by the discovery of its strong similarity in terms of kinematical features to $\alpha$ Cen (Innes 1926; Luyten 1925; Alden 1928), only recently it was demonstrated that Proxima is bound to the $\alpha$ Cen system (Kervella et al. 2017). The identification of such wide, physically bound binaries is relevant for our understanding of cloud fragmentation and star formation processes. El-Badry \& Rix (2019) show that 100-200 (au) is the separation range below which binaries are more likely to have formed via fragmentation of individual gravitationally unstable disks rather than through turbulent core fragmentation.

\footnotetext{
* Based on observations collected at the European Southern Observatory at Paranal, under program 103.C-0200(A), and archive data from 074.C-0037(A) and 082.C-0390(A).
}

Wider binaries, on the other hand, appear to have formed during the dissolution phase of young star clusters as they cannot be explained through the star formation process or by dynamical interactions in the field (Kouwenhoven et al. 2010; Moeckel \& Clarke 2011). These wider binaries are expected to exist at a young age and subsequently to be destroyed by dynamical interactions with individual stars and giant molecular clouds (Weinberg et al. 1987; Caballero 2009; Jiang \& Tremaine 2010). There is evidence that such systems exist with young ages (e.g. the AU Mic/AT Mic system with separation $0.23 \mathrm{pc}$ ), although the fact that young stars are typically members of loose groups or associations leaves ambiguity between bound systems and comoving objects (Alonso-Floriano et al. 2015). Generally, wide pairs are expected to exist with separations up to $\sim 1 \mathrm{pc}$ (Chanamé \& Gould 2004; Quinn et al. 2009; Tian et al. 2019).

The study of wide binaries additionally allows us to put constraints on the dynamical environment of a star, which impacts the evolution of the disk and planetary system around it. On a long (Gyr) timescale, even a distant, low-mass companion 
(hundreds of astronomical units) could shape a planetary system through the Kozai mechanism, increasing the eccentricity of the orbit of the existing planets (Kaib et al. 2013) and possibly producing hot-Jupiters (Wang et al. 2017). The case of substellar companions at wide separation around stars that host planets or brown dwarfs at separations close enough to be formed in the circumstellar disk is of great interest. A recently discovered example is the $\epsilon$ Ind system, with a Jupiter-like planet in a slightly eccentric orbit (Feng et al. 2019) and a close pair of brown dwarfs at very wide separation (Scholz et al. 2003; King et al. 2010). Such relatively old systems provide us with benchmark cases for studying the formation of gas giant planets and brown dwarfs.

Physical companions can also be used to improve the determination of system age (Ghez et al. 1993; Duchéne et al. 2007; Connelley et al. 2008; Tobin et al. 2016), especially when the central stars bear limited sensitivity to age indicators - as may be the case for both young and old stars, early and late type (e.g., see Garcés et al. 2011; Chanamé \& Ramírez 2012). Depending on the mass and evolutionary stage, companions can provide an opportunity to derive the ages of systems thanks to the determination of lithium in their spectra, gravity-sensitive spectral features (such as $\mathrm{NaI}, \mathrm{KI}, \mathrm{RbI}$ ), magnetic activity, and placement on the color-magnitude diagram (CMD). If the candidate is only a member of the stellar association and not physically bound to its associated star, there is still valuable information to extract from its physical parameters. Identifying new members of an association contributes to a better determination of age and chemical composition of the association. The discovery of multiple systems in young associations is of great value because it offers the opportunity to study objects of different mass whose kinematics still reflects the initial conditions of their parental cloud and is likely unaffected by interactions with nearby bodies.

The synergy between Gaia DR2 (Gaia Collaboration 2018) and the Spectro-Polarimetric High-contrast Exoplanet REsearch (SPHERE) instrument represents an important step forward for the identification of wide companions. Gaia has started to provide a wealth of information for the comprehensive identification of bound stellar companions (Andrews et al. 2017). The recent DR2 release allows us to further boost this kind of search, thanks to the extension to much fainter magnitudes, the increased accuracy of parallaxes and proper motion, and the availability of high-quality photometry and color information and even radial velocities for a subsample of targets. The sensitivity of Gaia is high enough to allow the identification of brown dwarf companions at wide separations from young stars in the closest star-forming regions and young associations (age $<20 \mathrm{Myr}$, dist $\leq 150 \mathrm{pc}$ ). In association, SPHERE, an extreme-AO instrument installed at the VLT since 2014 (Beuzit et al. 2019), is providing fascinating results on the detection and characterization of planetary mass companions, with some cases even being observed in the process of formation (Keppler et al. 2018). This instrument is also providing an unprecedented view of circumstellar disks (Garufi et al. 2017) thanks to a variety of instrument modes and its superb image quality as well as improved spatial resolution. Most of the targets observed with SPHERE are young stars (Desidera et al. 2020), because of the increased brightness of substellar objects at young ages and typical lifetimes of circumstellar disks.

In this context, we performed a pilot program, identifying wide companion candidates around SPHERE-GTO targets with directly imaged substellar companions or spatially resolved disks. We selected five candidates for this program and the current paper presents the spectroscopic characterization of four of them. An early result of this program, the identification and characterization of a new probable wide companion of GQ Lup was presented in Alcalá et al. (2020). Here we present additional physical parameters of this target as a complement to the addressed letter and characterize the other four candidates by means of intermediate-resolution VLT/X-shooter spectroscopy. The outline of the paper is as follows. In Sect. 2, the process of target selection exploiting SPHERE-GTO and Gaia DR2 capabilities is explained. In Sect. 3, we describe the spectroscopic observations, data reduction, and the methods of analysis used for the determination of stellar parameters. We present the results of our study in Sect. 4 and discuss the configuration of these systems in the framework of current formation theories in Sect. 5 .

\section{Target selection}

The five wide companion candidates were selected based on Gaia DR2 and SPHERE data. Possible wide companions to SPHERE-GTO targets were searched for in the Gaia DR2 catalog, and several tens of candidates were found based on their parallaxes and proper motions. Follow-up spectroscopic observations are therefore mandatory in order to confirm the young age of the proposed targets (high levels of magnetic activity, low-gravity spectral features), study the occurrence of accretion, and determine the effective temperature. While we expect at least some of our candidates to be gravitationally bound to their central stars, they might also be physically unbound to the selected system. In the latter case, our targets are either members of the association they are selected from and are incidentally comoving with the central star, or, in the worst scenario, they are field objects appearing in the association by chance. However, in order to confirm two objects as a physical pair we need more accurate measurements of the radial velocities of both the central stars and their probable wide companions. Also, for some of the targets, further observations of the close, directly imaged companions will allow us to take into account their impact on the kinematic properties of the central stars. Below, we provide a brief description of the individual targets and their specific features. For a summary of the physical properties of the central stars see Table 1, and for the kinematic properties of the objects see Table 5. To the best of our knowledge, the central stars selected in this work and their wide companion candidates are not listed in the previously investigated wide binaries in open clusters (see, e.g., Kraus \& Hillenbrand 2007; Deacon \& Kraus 2020).

For convenience, we introduce abbreviations for the names of our targets: 2MASS J18152222-3249329 is referred to as 2MASS J1815-3249, 2MASS J15174874-3028484 as 2MASS J1517-3028, 2MASS J13242119-5129503 as 2MASS J13245129, 2MASS J14571503-3543505 as 2MASS J1457-3543, and 2MASS J15491331-3539118 as 2MASS J1549-3539.

2MASS J1815-3249 is a possible additional member of the V4046 Sgr system. The central object is a close binary composed of two young stars orbiting with a period of 2.4 days and still accreting matter from a gas-rich circumbinary disk (Stempels \& Gahm 2004; Rosenfeld et al. 2013; D'Orazi et al. 2019). It is also the oldest Classical T Tauri star to date with a spectral type (SpT) earlier than M5 (the expected age of the system is $24 \mathrm{Myr}$ according to isochrones and its membership to the $\beta$-Pictoris Moving Group; Torres et al. 2008). The wide companion GSC 7396-0759, instead, hosts a recently discovered debris disk (Sissa et al. 2018) and is not accreting. The 
Table 1. Physical parameters of the central stars.

\begin{tabular}{lcccccccc}
\hline \hline Name & $\begin{array}{c}\text { Distance } \\
(\mathrm{pc})\end{array}$ & Association & SpT & $\begin{array}{c}T_{\text {eff }} \\
(\mathrm{K})\end{array}$ & $\begin{array}{c}A_{v} \\
(\mathrm{mag})\end{array}$ & Wide companion & $\begin{array}{c}\text { Separation } \\
\left({ }^{\prime \prime}\right)\end{array}$ & $\begin{array}{c}\text { Separation } \\
(\mathrm{au})\end{array}$ \\
\hline V4046Sgr & 72.4 & $\beta$-Pictoris MG & K5/K7 & $4370 / 4100$ & $0^{(b)}$ & 2MASS J1815-3249 & 901 & 65232 \\
HIP 74865 & 123.53 & Sco-Cen (UCL) & F3V & 6720 & 0 & 2MASS J1517-3028 & 90 & 11118 \\
HIP 65426 & 109.21 & Sco-Cen (LCC) & A2V & 8840 & 0 & 2MASS J1324-5129 & 142 & 15508 \\
HIP 73145 & 133.65 & Sco-Cen (UCL) & A2IV & 8840 & 0 & 2MASS J1457-3543 & 280 & 37422 \\
GQ Lup & 151.82 & Sco-Cen (Lupus I) & K7V & 4070 & $0.7^{(c)}$ & 2MASS J1549-3539 & 16 & 2429 \\
\hline
\end{tabular}

Notes. ${ }^{(a)}$ Distances are calculated based on the parallaxes of the objects reported in Gaia DR2 catalog. ${ }^{(b)}$ (Stempels \& Gahm 2004), ${ }^{(c)}$ (Alcalá et al. 2017).

discovery of another component of the system of slightly lower mass than GSC 7396-0759 would provide additional information on disk dispersal timescales. The new candidate also has an X-ray counterpart from XMM (source 3XMM J181522.2324932 from Rosen et al. 2016), suggesting it is an active star. Information on the separation of the object from the central star and its magnitudes is included in Tables 1 and 6, respectively.

2MASS J1517-3028 is a possible wide companion of HIP 74865. It is a member of the Sco-Cen association, has a brown dwarf companion at small separation $\left(0.13^{\prime \prime}=16 \mathrm{au}\right)$, and was discovered with Sparse Aperture Masking observations (Hinkley et al. 2015) and was recently characterized with SPHERE (Cantalloube et al., in prep.). The expected mass of the new candidate is about $0.17 M_{\odot}$ if coeval to the central star.

2MASS J1324-5129 is a possible, very wide companion of HIP 65426, and hosts a planetary-mass companion recently discovered with SPHERE at about 90 au (Chauvin et al. 2017). The system is part of the Sco-Cen association and its expected mass is about $25 M_{\text {jup }}$ if the target is coeval to HIP 65426.

2MASS J1457-3543 is a possible comoving object to HIP 73145. HIP 73145 is an A2IV star with an age of $\sim 15 \mathrm{Myr}$. This star was confirmed to be a member of the Scorpius-Centaurus association (Rizzuto et al. 2011) and a debris disk with concentric rings around it was recently spatially resolved by Feldt et al. (2017). The large projected separation $\left(280^{\prime \prime}, 38000 \mathrm{au}\right)$ and the kinematic properties of the wide companion candidate compared to those of the central star lower the chances of them being physically associated. However, the excess noise of 0.993 mas with a significance of 3.29 reported for 2MASS J1457-3543 in Gaia DR2 may imply some bias in the astrometric parameters. This target is the faintest object in our sample $(G=18.52, K s=13.11)$ and has very red colors.

2MASS J1549-3539 is a highly probable wide companion of GQ Lupi, and is a very young star (age $\sim 2 \mathrm{Myr}$ ) in the Lupus cloud according to Alcalá et al. (2020). GQ Lupi hosts a brown dwarf (BD) companion (GQ Lup B; mass about 20-40 $M_{\text {jup }}$ ) at $0.7^{\prime \prime}$ and has a disk spatially resolved by ALMA (MacGregor et al. 2017). The BD companion is a strong accretor, indicating the presence of a disk around it (Zhou et al. 2014). The full characterization of 2MASS J1549-3539 was carried out by Alcalá et al. (2020) and their results are presented again here for comparison with other candidates. For the sake of completeness, we report the equivalent widths (EWs) of additional lines that are absent from this latter paper. Lazzoni et al. (2020) spatially resolved the disk around the star exploiting HST archive images.

\section{Observations and methods}

\subsection{Observations}

$\mathrm{X}$-shooter spectra provide us with a wide range of wavelengths, which enables us to perform spectral classification and accretion evaluation as suitable indicators are spread in different spectral regions. For details of the instrument description, the reader is referred to Vernet et al. (2011). X-shooter also enables us to determine the radial velocity of objects within a precision range of $2-5 \mathrm{~km} \mathrm{~s}^{-1}$ (Frasca et al. 2017) and is therefore useful for confirming or ruling out the physical association of the candidates with their parent stars. Four of our targets were observed through the $1^{\prime \prime} 0,0 \prime \prime 9$, and $0 \prime \prime 9$ slits for one or two cycles based on their $G$ and $J$ band magnitudes. Among these four, for the three fainter objects we performed two cycles of ABBA nodding mode. These three targets are 2MASS J1324-5129, 2MASS J1457-3543, and 2MASS J1549-3539 with $G$ magnitudes $18.71,18.52$, and 18.37 respectively. As measuring line fluxes with a precision better than $20 \%$-at least in the visible (VIS) and near-infrared (NIR) armswas a necessity for achieving our aims, a total on-source execution time of $1.2 \mathrm{hr}$ was requested for the aforementioned objects. For 2MASS J1517-3028, which is brighter than the other three targets, one cycle of ABBA nodding mode was performed with a total execution time equal to $1 \mathrm{hr}$. The corresponding $G$ magnitude for this latter candidate is 15.80 . For a summary of the color magnitudes of our targets see Table 6 .

2MASS J1815-3249 is the brightest among our five targets with a $G$ magnitude of 13.60 . We therefore decided to observe it with the highest resolution offered by X-shooter, adopting the slits $0 \prime \prime 5,0 \prime .4$, and $0 \prime \prime 4$, amounting to an execution time of 0.7 hr. For all five targets, short exposures (of $\sim 10 \%$ of the time allocated to the nodding mode) were performed before science observations through $55^{\prime} 0$ slits in order to obtain a more accurate flux calibration and to compensate the slit losses; the slit-loss correction factor (SLCF) for each arm is reported in Table 2. These short exposures in stare mode were incorporated in the same observation block (OB) consisting of the nodding mode in order to minimize the overheads. For each object, telluric standards were observed exploiting the same nodding strategy as for the targets in order to minimize noise and cosmetics, with an airmass as close as possible to the targets' airmass for telluric correction purposes. The reported airmass and seeing in Table 2 are averaged over the observation periods for each arm. All targets were observed at parallactic angle.

\subsection{Data reduction}

Data reduction was carried out using EsoReflex version 2.9.1 (Freudling et al. 2013) and through X-shooter pipeline version 
Table 2. Observation policies information table.

\begin{tabular}{|c|c|c|c|c|c|c|c|c|c|c|}
\hline Name & $\begin{array}{c}\text { Date } \\
\text { (yyyy-mm-dd) }\end{array}$ & $\begin{array}{c}\alpha(\mathrm{J} 2000) \\
(\mathrm{h}: \mathrm{m}: \mathrm{s})\end{array}$ & $\begin{array}{c}\delta(\mathrm{J} 2000) \\
(\mathrm{d}: \mathrm{m}: \mathrm{s})\end{array}$ & $\begin{array}{c}G \\
(\mathrm{mag})\end{array}$ & $\begin{array}{c}\text { Nodding slits } \\
\left({ }^{\prime \prime}\right)\end{array}$ & $\begin{array}{c}\text { Exposure time } \\
(\mathrm{sec})\end{array}$ & $\begin{array}{l}\text { Seeing } \\
\left({ }^{\prime \prime}\right)\end{array}$ & $\begin{array}{c}T_{\text {tot }} \\
\text { (hour) }\end{array}$ & SLCF & Airmass \\
\hline 2MAS & $19-05-24$ & 181522.23 & -324933.07 & 13.6 & $0^{\prime \prime} .5,0^{\prime \prime} .4,0^{\prime \prime} .4$ & $2 \times / 450 / 400 / 450$ & $0.955 / 0.875 / 0.805$ & 0.7 & $1.35 / 1.35 / 1.35$ & 1.01 \\
\hline 2MASS J1517-3028 & $2019-05-24$ & 151748.75 & -302848.42 & 15.8 & $1^{\prime \prime} 0,0,0^{\prime \prime} 9,0^{\prime \prime} 9$ & $2 \times / 800 / 750 / 800$ & $1.01 / 1.01 / 1.01$ & 1 & $1.5 / 1.5 / 1.6$ & 1.09 \\
\hline J1324-5129 & & 132421.18 & -512950.39 & 18.71 & $1^{\prime \prime} .0,0 \prime \prime 9,0^{\prime \prime} 9$ & $4 \times / 800 / 750 / 800$ & $0.905 / 0.905$ & 1.5 & $1.47 / 1.34 / 1.45$ & 1.21 \\
\hline 2MASS J1457-3543 & 2019-05-30 & 145715.03 & -354350.64 & 18.52 & $1^{\prime \prime} .0,0 \prime \prime 9,0^{\prime \prime} 9$ & $4 \times / 800 / 750 / 800$ & $0.66 / 0.66 / 0.66$ & 1.5 & $1.22 / 1.22 / 1.39$ & 1.18 \\
\hline 2MASS J1549-3539 & 2019-06-25 & 154913.30 & -353911.79 & 18.37 & $1^{\prime \prime} .0,0^{\prime \prime} \cdot 9,0^{\prime \prime} 9$ & $4 \times / 800 / 750 / 800$ & $1.98 / 1.965 / 1.965$ & 1.5 & $2.6 / 2.2 / 2.0$ & 1.05 \\
\hline
\end{tabular}

Notes. Nodding slits, the allocated exposure time to each arm, seeing, and SLCF are reported in order for UVB, VIS, and NIR wavelengths. The number of single observations is presented as a multiplication factor. The column allocated to $T_{\text {tot }}$ presents the total execution time for the targets.

3.3.5 (Modigliani et al. 2011). This software has automated the data-reduction process and produces the spectra corresponding to each X-shooter arm separately using the raw data files. Reducing a science frame requires the following calibration frames: pinhole arc-lamp format-check frames, biases and dark frames for UVB and VIS arms, pinhole continuum-lamp order definition, flat fields, multi-pinhole arc-lamp, and flexure compensation frames. It is noteworthy that flexure compensation frames are optional for more accurate wavelength calibrations. For a detailed description of how each module operates, the reader is referred to the X-shooter pipeline manual published by ESO.

\subsection{Analysis method}

The spectra extracted from EsoReflex were then analyzed by means of the Image Reduction and Analysis Facility (IRAF) ${ }^{1}$ and our own software developed in IDL environment ${ }^{2}$. Telluric lines were removed using the IRAF telluric package. The flux was corrected for slit losses by dividing the stare mode observations by nodding mode observations. At this stage, the flux was compared with the available photometric data in the literature and good agreement between the two was achieved for each target, within the errors of photometry and flux calibration of the spectra, which is on the order of $15-20 \%$. The IRAF environment was also used to obtain the physical parameters of each target: SpT, effective temperature $\left(T_{\text {eff }}\right)$, radial velocity $(R V)$, and EWs of spectral lines. We also determined the same physical parameters plus the RV, the projected rotational velocity $(v \sin i)$, and the surface gravity $(\log g)$ with the version of the code ROTFIT adapted to X-shooter spectra (Frasca et al. 2017). We refer the reader to this latter paper for details on this analysis code. In this way, we were able to double-check or revise our final results. In the following paragraph, we give a brief explanation of the general procedure we use for characterizing the targets. The results from ROTFIT are reported separately in Table 4.

To estimate SpTs for our objects, we first compared the spectrum of each object with a library of stars and brown dwarfs with known parameters observed formerly by X-shooter (Manara et al. 2013, 2017). As the spectra are taken with the same instrument and setup, their direct comparison allows us to evaluate SpT and extinction. We then took the average over the spectral indices reported in Riddick et al. (2007) to quantitatively determine the SpTs of our objects. The fitting indices in this paper all belong to the VIS portion of the spectrum,

\footnotetext{
1 IRAF is distributed by the National Optical Astronomy Observatory, which is operated by the Association of the Universities for Research in Astronomy, inc. (AURA) under cooperative agreement with the National Science Foundation.

2 IDL (Interactive Data Language) is a registered trademark of Harris Corporation.
}

with an error of 0.5 subclasses. Once the SpT was determined, the effective temperature was derived from the calibration of Pecaut \& Mamajek (2013) ${ }^{3}$. We used the bolometric correction relation proposed by these latter authors to evaluate the luminosity in both $V$ and $J$ bands and radius of candidates according to their observed parallax and magnitudes. The luminosity of the objects included in Table 3 was calculated by integrating the flux of X-shooter spectra and the fitted BT-Settl model, which was double-checked by the luminosities obtained in $V$ and $J$ bands as explained earlier. Radial velocities (RVs) were determined by cross-correlating portions of the targets' VIS spectra free from emission lines with a template spectrum with the same $\mathrm{SpT}$ and low $v \sin i$. All the RVs reported in this paper are extracted from the visual part of the spectrum only, because it has the highest resolution and/or signal-to-noise ratio $(\mathrm{S} / \mathrm{N})$ compared to UVB and NIR spectra. The RVs are calculated with the IRAF task FXCOR and the code ROTFIT, which adopts synthetic BT-Settl spectra (Allard et al. 2012) as templates. The EWs are measured either by fitting a Gaussian to the lines if the line is sharp enough, or direct integration of the pixel values between two marked pixels. A considerable amount of extinction is not expected for any of our targets, except 2MASS J1549-3539. We adopt a zero extinction for cases in which unphysical negative extinctions or extinctions lower than the predicted error $(0.5$ mag) were achieved ${ }^{4}$.

Besides these two methods, for a final consistency check, we also fitted the spectrum of each target to the BT-Settl theoretical spectra models (Allard et al. 2012). These grids of stellar and substellar atmosphere models confirm our conclusions as they estimate the $T_{\text {eff }}$ and $\log g$ of our candidates. To evaluate the age and mass of the targets, we calculated their luminosity and radius first, and then used the available isochrones consistent with these parameters: MESA Isochrones and Stellar Tracks (MIST, Paxton et al. 2015; Choi et al. 2016; Dotter 2016) and stellar models generated by Baraffe et al. (2015). At this point, we could once again check the $T_{\text {eff }}$ and $\log g$ of our candidates. All the adopted stellar models have solar metallicities.

\section{Results}

In this section, we report the result of our analysis with IRAF and ROTFIT. The kinematic properties of GQ Lupi b are reported in Neuhäuser et al. (2008) and Schwarz et al. (2016). It is noteworthy that 2MASS J1815-3249 is a possible member of the $\beta$-Pictoris MG, while the other four candidates are located in various clouds of the Scorpius-Centaurus association (hereafter

http://www.pas.rochester.edu/ emamajek/EEM_dwarf_ UBVIJHK_colors_Teff.txt

$4 A_{v}$ is not determined by ROTFIT, which analyzes the continuumnormalized target spectrum. 
Table 3. Physical stellar parameters of candidates.

\begin{tabular}{|c|c|c|c|c|c|c|c|c|c|}
\hline Name & $\mathrm{SpT}$ & $\begin{array}{l}T_{\text {eff }} \\
(\mathrm{K})\end{array}$ & $\begin{array}{c}A_{v} \\
(\mathrm{mag})\end{array}$ & $\begin{array}{c}\mathrm{RV} \\
\left(\mathrm{km} \mathrm{s}^{-1}\right)\end{array}$ & $\begin{array}{l}L_{\star} \\
\left(L_{\odot}\right)\end{array}$ & $\begin{array}{c}R_{\star} \\
\left(R_{\odot}\right)\end{array}$ & $\begin{array}{c}M_{\star} \\
\left(M_{\odot}\right)\end{array}$ & $\begin{array}{l}\text { Age } \\
\text { (Myr) }\end{array}$ & $\log g$ \\
\hline & & & U & & & & 0.3 & & 4.95 \\
\hline $2 \mathrm{~N}$ & - & & 0 & $1.2 \pm$ & 0.0 & 0.47 & 0.11 & 7.9 & 4.13 \\
\hline $2 \mathrm{MA}$ & 5 & 2710 & 0 & $15.3 \pm$ & 0.0024 & 0.22 & 0.04 & 18.2 & 4.35 \\
\hline & & 2500 & 0 & & & 0.24 & 0.02 & 14.09 & 4.00 \\
\hline 2MASS J1549-3539 & $\mathrm{M} 4 \pm 0.5$ & 3190 & $1.0 \pm 0.5$ & $-1.3 \pm 4.0$ & $0.07^{(a)}$ & 0.21 & 0.15 & 2 & 3.78 \\
\hline
\end{tabular}

Notes. Except for 2MASS J1815-3249, mass, age, and $\log g$ are selected from the closest matches offered by Baraffe et al. (2015) models. The same quantities are extracted from MIST isochrones for 2MASS J1815-3249. ${ }^{(a)}$ This object is sub-luminous. The value reported here is the object's corrected luminosity obtained by Alcalá et al. (2020).

Table 4. ROTFIT results acquired for the targets. The estimated ages are concluded from Baraffe et al. (2015) models.

\begin{tabular}{lccccc}
\hline \hline Name & $\begin{array}{c}T_{\text {eff }} \\
(\mathrm{K})\end{array}$ & $\log g$ & $\begin{array}{c}\mathrm{RV} \\
\left(\mathrm{km} \mathrm{s}^{-1}\right)\end{array}$ & $\begin{array}{c}v \sin i \\
\left(\mathrm{~km} \mathrm{~s}^{-1}\right)\end{array}$ & $\begin{array}{c}\text { Age } \\
(\mathrm{Myr})\end{array}$ \\
\hline 2MASS J1815-3249 & $3562 \pm 30$ & $4.68 \pm 0.14$ & $-20.1 \pm 2.0$ & $<8.0$ & $>150$ \\
2MASS J1517-3028 & $3077 \pm 22$ & $4.49 \pm 0.21$ & $1.4 \pm 2.4$ & $26 \pm 6$ & $7.4 \pm 0.5$ \\
2MASS J1324-5129 & $2646 \pm 50$ & $4.0 \pm 0.1$ & $17.9 \pm 3.0$ & $<8.0$ & $16 \pm 2.2$ \\
2MASS J1457-3543 & $2635 \pm 80$ & $4.30 \pm 0.17$ & $8.0 \pm 7.8$ & $62.0 \pm 10.0$ & $17.75 \pm 4.15$ \\
2MASS J1549-3539 & $3230 \pm 101$ & $3.74 \pm 0.23$ & $-2.0 \pm 2.8$ & $13.0 \pm 6.0$ & $2.75 \pm 0.75$ \\
\hline
\end{tabular}

Sco-Cen). Sco-Cen is the closest OB association to the Sun, consisting of three main clouds and some areas with a diffuse kinematical population (Damiani et al. 2019, and references therein). The central stars of these candidates are acknowledged as members of the association according to Rizzuto et al. (2011). For all the candidates, we also checked the astrometric excess noise and blue-red (BP-RP) excess factors reported by Gaia DR2 to check for signs of binarity. None of the targets show a significant $B P$ $R P$ excess factor ( $\sim 2-3$ addressing a source heavily affected by noise or a highly populated region), while a significant astrometric excess noise $(>1)$ was reported for 2MASS J1324-5129 and 2MASS J1457-3543. This latter has been confirmed by a considerable value of astrometric-excess-noise-sigma $(>2)$ reported for the two objects. The spectra acquired for these two candidates are rather noisy compared to the other three and were more challenging to analyze.

The excess noise takes the modeling errors into account (Lindegren et al. 2012) and a value close to zero indicates an astrometrically well-behaved single source. However, not all sources behave exactly as the best-fitting standard astrometric model. For example, mid-late M-type members of the Upper Scorpius sub-association have high astrometric excess noise because they are intrinsically faint and the five-parameter fit is therefore imperfect. We therefore investigated the members of Upper Centaurus-Lupus (UCL) and Lower Centaurus-Crux (LCC) studied by Damiani et al. (2019) to understand whether or not the members of these two sub-associations, to which three of our candidates belong, also show significant astrometric excess noise. Our studies indicate that among the upper main sequence (UMS) and pre main sequence (PMS) members of LCC, collectively, 75 out of 90 members have a significant astrometric excess noise, and this number is 597 out of 697 members for UCL. We also calculated the renormalised unit weight error (RUWE) associated to each source which is more reliable as an indicator of the goodness-of-fit statistic than astrometric excess noise (Lindegren et al. 2018).
For two of our targets, namely 2MASS J1815-3249 and 2MASS J1457-3543, we could not measure the $E W_{\text {Li I }}$ because of non-detection of the lithium line, and therefore we only report an upper limit. For this purpose, a three-sigma upper limit on the flux of the lithium line is calculated based on the spectra of the objects (Cayrel 1988):

$d E W=3 \times 1.06 \sqrt{(F W H M) d x} /(S / N)$,

in which FWHM is the full width at half maximum, $\mathrm{S} / \mathrm{N}$ is the signal-to-noise ratio, and the bin size (dx) is $0.2 \AA$ for the VIS arm. In Fig. 8b, we indicate the upper limits of $E W_{\mathrm{Li} \text { I }}$ for the aforementioned candidates as downward arrows.

\subsection{MASS J1815-3249}

The spectrum of 2MASS J1815-3249 (Fig. 1a) displays clear features of an M2-3 type star, which is reported in Table 3 in addition to its other stellar parameters. For this star, the measured $v \sin i$ with ROTFIT is lower than $8 \mathrm{~km} \mathrm{~s}^{-1}$. As the minimum detectable $v \sin i$ at this resolution is $8 \mathrm{~km} \mathrm{~s}^{-1}$ (Frasca et al. 2017), we report only an upper limit of $8 \mathrm{~km} \mathrm{~s}^{-1}$. The Li I line was not detected in the spectrum. However, this is not conclusive for determining the age of the object as no lithium is expected for a M2-3 star even at the young age of $\beta$ Pictoris MG (Fig. 8b). Regarding the chromospheric activity, the $\mathrm{H}_{\alpha}$ line was found to be in absorption and moderate Ca II-IRT activity was detected. Regarding the physical association with the central star, V4046 Sgr was measured to have a $\mathrm{RV}=-6.94 \pm 0.16$; its center of mass RV was obtained through orbital solution by Quast et al. (2000); and its low-mass companion, GSC 7396-00759, has $\mathrm{RV}=-6.10 \pm 0.5$ (Sissa et al. 2018). The values of RV derived by us for 2MASS J1815-3249 with the two adopted procedures (Tables 3 and 4) agree with each other within the errors, but are significantly different from the barycentric velocity of V4046 Sgr and the RV of GSC 7396-00759. This argues against a physical association of this star with the stellar system; for a summary 


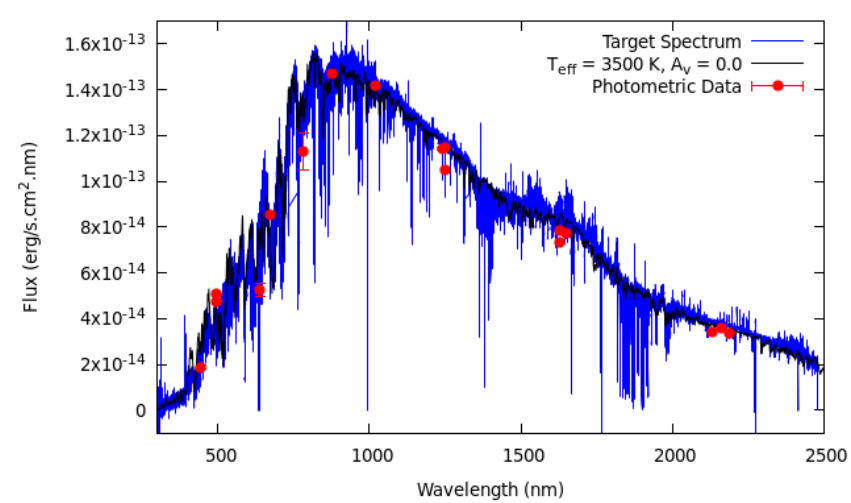

(a)

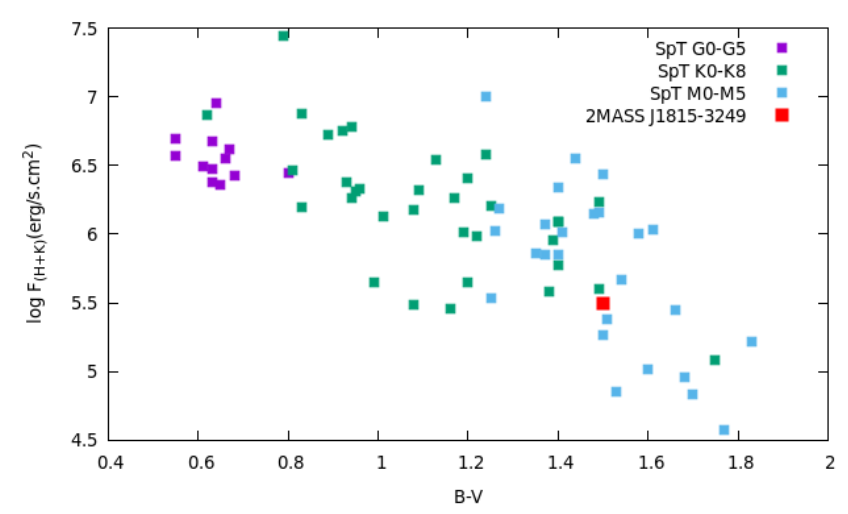

(b)

Fig. 1. Panel a: spectrum of 2MASS J1815-3249 (blue) together with a BT-Settl model (black) fitted to the spectrum assuming zero extinction. Panel $b$ : total excess emission in Ca II $\mathrm{H}$ and $\mathrm{K}$ vs. color index B-V of objects studied by López-Santiago et al. (2010) and Martinez-Arnáiz et al. (2011) including 2MASS J1815-3249, shown as a red square.

of their collective kinematic properties see Table 5. The chromospheric activity is evident as a small filling in the cores of Ca IIIRT lines and emission above the continuum level in the cores of the $\mathrm{Ca}$ II $\mathrm{H}$ and $\mathrm{K}$ lines. We measured the flux in the $\mathrm{H}$ and $\mathrm{K}$ lines, integrating the emission features arising from the subtraction of the inactive template produced by the BT-Settl spectrum with $T_{\text {eff }}=3500 \mathrm{~K}$. We compared the $\mathrm{H}$ and $\mathrm{K}$ flux of $2 \mathrm{MASS}$ J1815-3249 with that of late-type active stars investigated by López-Santiago et al. (2010) and Martinez-Arnáiz et al. (2011). As can be seen in Fig. 1b, the position of our target is well below the upper branch of the very active, young M-type stars and lies in the region occupied by older objects (Age $\geq 150 \mathrm{Myr}$ ). An age older than that of the $\beta$-Pictoris MG is also suggested by the CMD presented in Fig. 8a, which exhibits 2MASS J1815-3249 on the MS branch, unlike the other young candidates discussed in this work, which lie significantly higher.

The X-ray luminosity as derived from the observed X-ray flux (Rosen et al. 2016) coupled with our adopted stellar parameters yields $\log \left(L_{X} / L_{\mathrm{bol}}\right)=-4.14$. This value is consistent with those of Hyades stars of similar colors, and well below those of members of younger clusters or groups such as the Pleiades and the $\beta$-Pictoris MG (Song et al. 2008), indicating a most probable age of several hundred million years.

The age inconsistency with the $\beta$-Pictoris MG, which is supported by the position of 2MASS J1815-3249 on the CMD, a low level of magnetic activity, and the absence of lithium absorption, together with the discrepant RV with respect to that of the central star imply that this target is not a legitimate member of the group and is significantly older than expected.

\subsection{MASS J1517-3028}

2MASS J1517-3028 has already been identified as a PMS member of UCL (Damiani et al. 2019). The assessed age for the UCL sub-association was reported to be $15 \pm 3 \mathrm{Myr}$ by Pecaut \& Mamajek (2016), and therefore we adopt the same value as a first guess for our target. We also assume the same age for the central star which is an F4V star; for a detailed study, the reader is referred to Pecaut et al. (2012). The physical stellar parameters and ROTFIT results derived from the X-shooter spectrum are in good agreement with each other, and are displayed in Tables 3 and 4, respectively. The BT-Settl synthetic template, which is overplotted on the X-shooter target spectrum in Fig. 2, confirms the values of $T_{\text {eff }}$ and $\log g$ derived with ROTFIT. The fluxes derived from literature photometry, which are overplotted as red dots in Fig. 2, support the flux calibration we performed. The EW of the lines relevant for assessing chromospheric activity are incorporated in Table 7. The EW of $\mathrm{H}_{\alpha}$ and other hydrogen lines in emission plus $E W_{\mathrm{Li}}$ indicate a young and active star, meeting our preliminary expectations. These values also demonstrate that the object is not actively accreting matter from a circumstellar disk, as none of the measured hydrogen lines are sufficiently intense or wide. The central star, HIP 74865, which we initially surmised to be physically bound with 2MASS $\mathrm{J} 1517-3028$, has an RV $=2 \pm 0.3$ (Chen et al. 2011) and for the companion we have RV $=1.4 \pm 2.4$. This confirms the two stars share a significant similarity in kinematic properties; see Table 5 for a summary of their kinematic properties. Based on the stellar parameters of 2MASS J1517-3028, Baraffe et al. (2015) models suggest an age of $7.4 \pm 0.5 \mathrm{Myr}$ for this object which is younger than the age of the UCL sub-association by a factor of two. In a recent paper by Asensio-Torres et al. (2019), who investigated an Upper Scorpius-Centaurus member and its companions, namely HIP 79124ABC, the same problem is discussed. Although the components of this stellar system are expected to be coeval, both of the low-mass companions were estimated to have half the age of the central star. Feiden (2016) also pointed out that cooler $\mathrm{K}$ and $\mathrm{M}$ stars located in young stellar associations are reported to be younger than hotter A, F, or G stars belonging to the same association by a factor of two. This problem is probably due to neglect of the magnetic fields in stellar evolution isochrones which in turn fail to reproduce the age of low-mass PMS stars accurately (Asensio-Torres et al. 2019; Feiden 2016). Therefore, by adopting the most appropriate stellar evolution models tailored for ages beyond $10 \mathrm{Myr}$, we expect 2MASS J1517-3028 to have an age consistent with UCL. All aspects considered, we conclude there is a high chance that this candidate is physically associated with HIP 74865, offering evidence of a new triple system. The projected separation of about $11100 \mathrm{au}$ also supports the idea of this configuration, indicating a physically bound system.

\subsection{MASS J1324-5129}

This target is situated in the LCC sub-association of Sco-Cen, with a projected separation of 15500 au from the central star, and has not yet been listed as a member of Sco-Cen in the literature. The Li I line was found to be in absorption and various hydrogen lines are in emission, as indicated in Table 7. The $\mathrm{H}_{\alpha}$ line is wider than expected as it is apparently contaminated by 
Table 5. Kinematic properties of our targets and their associated stellar systems.

\begin{tabular}{lcccc}
\hline \hline Name & $\begin{array}{c}\text { Parallax } \\
(\mathrm{mas})\end{array}$ & $\begin{array}{c}\mu_{\alpha} \\
\left(\mathrm{mas} \mathrm{yr}^{-1}\right)\end{array}$ & $\begin{array}{c}\mu_{\delta} \\
\left(\mathrm{mas} \mathrm{yr}^{-1}\right)\end{array}$ & $\begin{array}{c}\mathrm{RV} \\
\left(\mathrm{km} \mathrm{s}^{-1}\right)\end{array}$ \\
\hline 2MASS J1815-3249 & $13.12 \pm 0.054$ & $1.07 \pm 0.095$ & $-52.74 \pm 0.078$ & $-20.1 \pm 2.0$ \\
V4046Sgr & $13.81 \pm 0.064$ & $3.49 \pm 0.11$ & $-52.75 \pm 0.087$ & $-6.94 \pm 0.16$ \\
GSC 7396-00759 & $13.99 \pm 0.052$ & $3.08 \pm 0.10$ & $-52.64 \pm 0.08$ & $-6.10 \pm 0.5$ \\
\hline 2MASS J1517-3028 & $8.16 \pm 0.11$ & $-21.67 \pm 0.21$ & $-28.31 \pm 0.18$ & $1.4 \pm 2.4$ \\
HIP 74865 & $8.09 \pm 0.061$ & $-21.07 \pm 0.11$ & $-28.42 \pm 0.10$ & $2.0 \pm 0.3$ \\
\hline 2MASS J1324-5129 & $8.01 \pm 0.35$ & $-31.85 \pm 0.53$ & $-17.07 \pm 0.44$ & $15.29 \pm 5.75$ \\
HIP 65426 & $9.16 \pm 0.062$ & $-34.25 \pm 0.10$ & $-18.81 \pm 0.093$ & $12.2 \pm 0.3$ \\
\hline 2MASS J1457-3543 & $9.86 \pm 0.42$ & $-28.68 \pm 0.68$ & $-27.3 \pm 0.65$ & $8.0 \pm 7.8$ \\
HIP 73145 & $7.48 \pm 0.20$ & $-23.35 \pm 0.26$ & $-24.94 \pm 0.30$ & $3.8 \pm 1.6$ \\
\hline 2MASS J1549-3539 & $6.59 \pm 0.05(a)$ & $-14.81 \pm 0.97$ & $-21.95 \pm 0.65$ & $-2.0 \pm 2.8$ \\
GQ Lup & $6.59 \pm 0.05$ & $-14.26 \pm 0.01$ & $-23.6 \pm 0.07$ & $-3.6 \pm 1.3$ \\
GQ Lup B & $7.2 \pm 2.1$ & - & - & $2.0 \pm 0.4$ \\
\hline
\end{tabular}

Notes. For a discussion on the adopted kinematic properties of 2MASS J1549-3539, the reader is referred to Alcalá et al. (2020); we have adopted the parallax of the central star for 2MASS J1549-3539 because of its highly uncertain measured kinematic features as the only accreting object among the targets. ${ }^{(a)}$ Considering that the astrometric parameters of GQ Lup C are somewhat biased by illumination effects (due to the presence of a disk and likely outflows), the parallx of GQ Lup has been adopted for this target; see Alcalá et al. (2020) for more information.

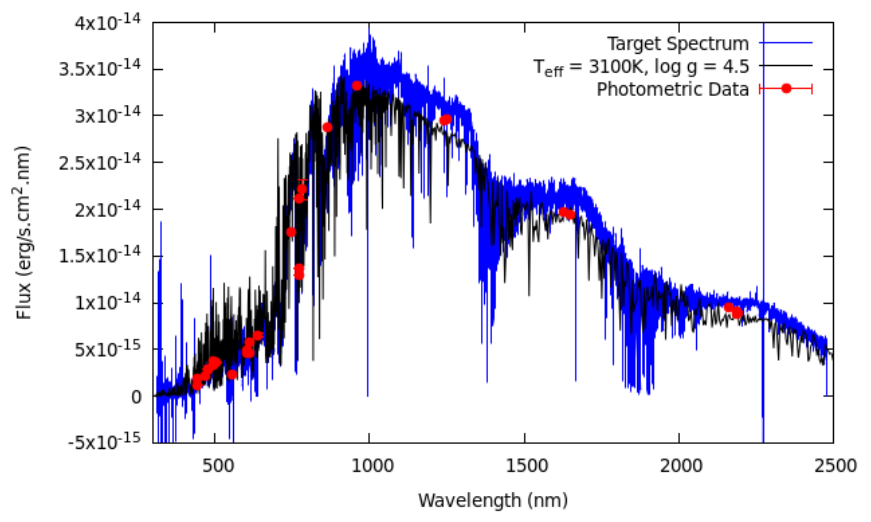

Fig. 2. Spectrum of 2MASS J1517-3028 (blue) together with the available photometric data (red dots) in the literature and the fitted BT-Settl model (black).

cosmic ray hits on the detector. The spectrum of this target shows features of an object with SpT of M6.5 \pm 0.5 subclasses (Fig. 3). Reylé (2018) evaluated the SpT of this object as M7 which is in good agreement with our estimation. We placed this object along with UMS and PMS members of LCC on a CMD (Fig. 4a) that shows 2MASS J1324-5129 is fainter than the studied sample by Damiani et al. (2019). As we pointed out at the beginning of this section, most of the objects in this sample have a significant astrometric excess noise. We therefore calculated only the RUWE of the LCC M-type members to make a comparison between the fainter objects of the sample and our target. As displayed in Fig. 4b, a few of these LCC members have a significant RUWE, above the threshold of 1.4, implying a nonsingle object or nonconsistent astrometric solution with the observations. The SpT of the members is estimated based on their $M_{\mathrm{G}}$ (Kiman et al. 2019). The RUWE of our object is 1.104 , which is not significantly higher than 1 and confirms the source is well-behaved.

Very recently, the RV of HIP 65426 and its companion HIP 65426 b were revisited (Petrus et al. 2020). According to these new evaluations, $\mathrm{RV}=12.2 \pm 0.3 \mathrm{~km} \mathrm{~s}^{-1}$ for HIP 65426 and $\mathrm{RV}=26 \pm 15 \mathrm{~km} \mathrm{~s}^{-1}$ for HIP $65426 \mathrm{~b}$. These values are well within the RV range we report for 2MASS J1324-5129 which

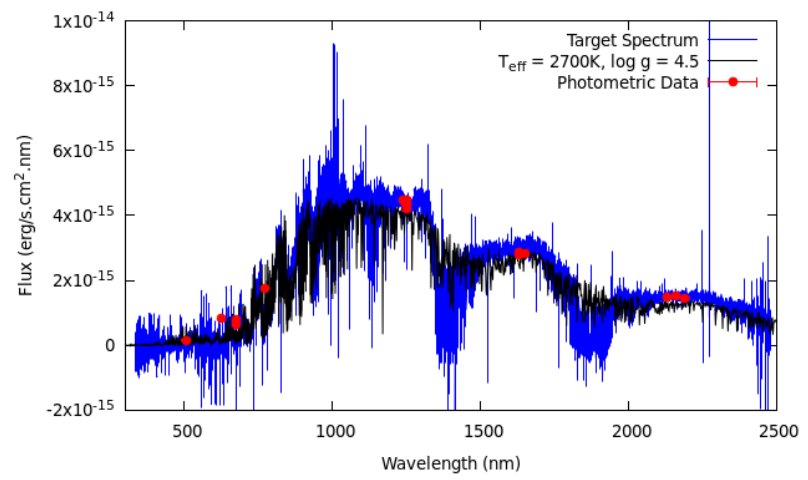

Fig. 3. Spectrum of 2MASS J1324-5129 (blue) with the photometric data (red dots) and the BT-Settl model (black) that confirms the estimated $T_{\text {eff }}$ and $\log g$.

is $15.29 \pm 5.75 \mathrm{~km} \mathrm{~s}^{-1}$ (although with large error bars). While displaying the typical features of an active young stellar object (YSO) and consistent RV with the central star, the slightly discrepant kinematic properties of 2MASS J1324-5129 and HIP 65426 argue against their physical association (see Table 5). As in 2MASS J1517-3028, 2MASS J1324-5129 is also not accreting matter from the central star according to Fig. 7. The position of 2MASS J1324-5129 on the CMD in Fig. 8a confirms its status as a PMS star. We checked the membership of this target through BANYAN $\Sigma$ (Gagné et al. 2018) and the probability of 2MASS J1324-5129 being a member of LCC is above 94\%. We therefore conclude that 2MASS J1324-5129 is a new PMS member of LCC. The age of $16.0 \pm 2.2 \mathrm{Myr}$ that we derive from the HR diagram and the Baraffe et al. (2015) evolutionary tracks is in perfect agreement with the age reported by Pecaut \& Mamajek (2016) for the LCC sub-association $(16.0 \pm 2.2 \mathrm{Myr})$. The mass that we derive with the same models, $M_{\star}=0.04 M_{\odot}$, confirms 2MASS J1324-5129 as a substellar object.

\subsection{MASS J1457-3543}

2MASS J1457-3543 is situated in UCL sub-association in Sco-Cen, the same as 2MASS J1517-3028, and has not been 


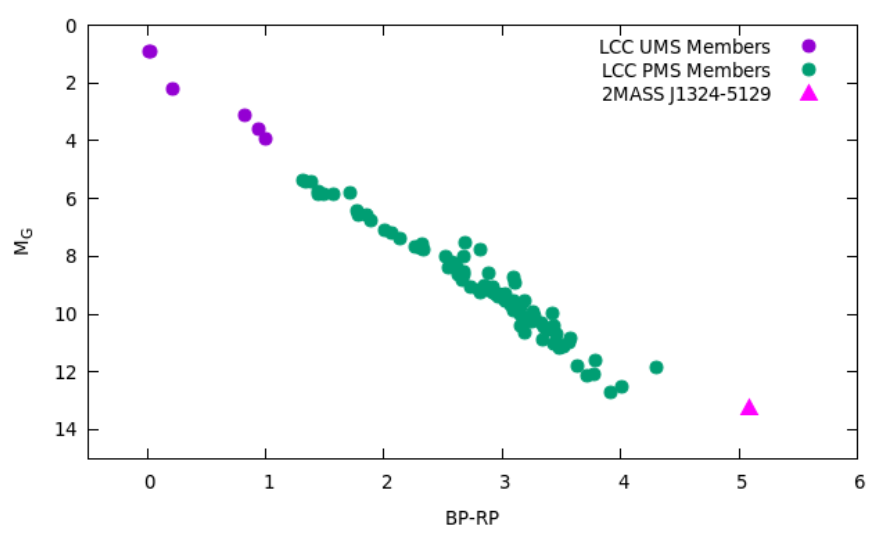

(a)

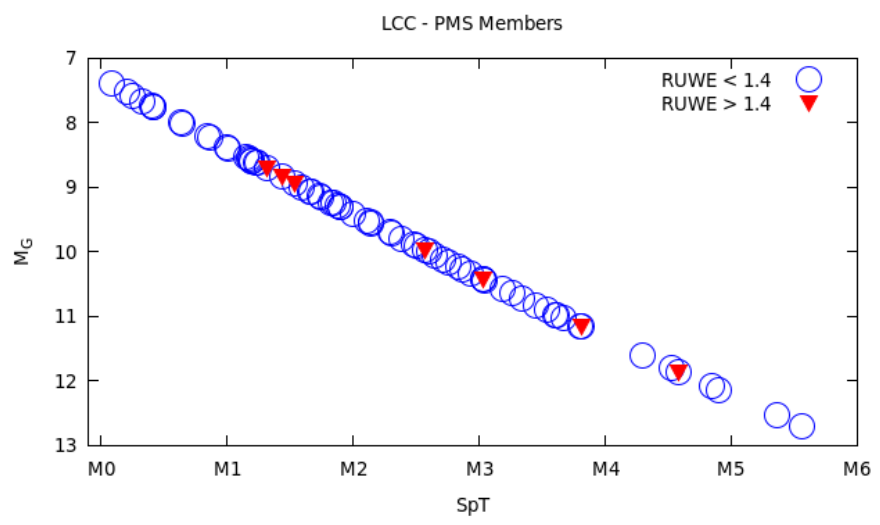

(b)

Fig. 4. Panel a: UMS and PMS members of LCC (Damiani et al. 2019) with our target in the same sub-association, 2MASS J1324-5129. Panel $b$ : RUWE of M-type members of LCC. The blue circles display members with RUWE indices below the threshold of RUWE $\sim 1.4$ while the red triangles represent members with RUWE $>1.4$ which refer to nonsingle or problematic objects for the astrometric solution.

confirmed as a member of the association in the literature. As discussed above, a physical association with HIP 73145 at $280^{\prime \prime}$ is unlikely, because of the slightly discrepant kinematic parameters. In order to have a full comparison of the kinematics, we derived the RV of the central $\operatorname{star}^{5}$ using the reduced FEROS and HARPS spectra of HIP 73145 available on ESO archive ${ }^{6}$.

According to Riddick et al. (2007) SpT indices, 2MASS J1457-3543 is an M8 ( \pm 0.5 subclasses) dwarf. Clear signs of activity, such as Balmer emission lines, are present in its spectrum. The kinematic properties of the target are compatible with Sco-Cen membership (with its UCL membership probability higher than $97 \%$ according to BANYAN). However, there is one crucial element missing in the spectrum of this target considering the general expectations from a Sco-Cen object of this spectral type: $\mathrm{Li}$ I. This nondetection could be due to the fast rotational velocity $v \sin i=62.0 \pm 10 \mathrm{~km} \mathrm{~s}^{-1}$ of this target or the low $\mathrm{S} / \mathrm{N}$ $(\sim 5)$ in that region. In this scenario, which is the most likely explanation, 2MASS J1457-3543 is a member of UCL with an estimated age of $17 \mathrm{Myr}$ according to Baraffe et al. (2015) models. We estimate an upper limit of $d E W_{\mathrm{Li} \text { I }}=390 \mathrm{~m} \AA$ for this

\footnotetext{
5 The RV quoted in SIMBAD for HIP 73145 at the time of writing is not a true measurement, but rather the kinematic RV expected for a member of the association as derived in Madsen et al. (2002).

6 archive.eso.org
}

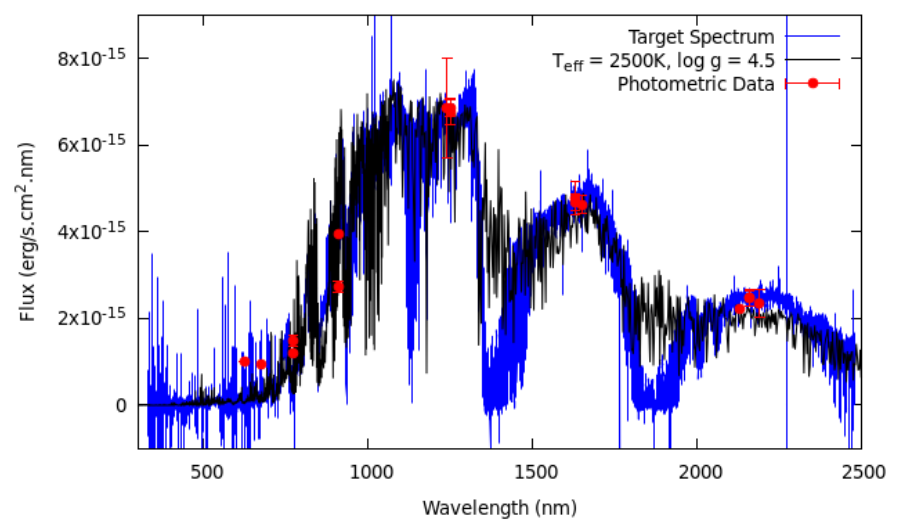

Fig. 5. Spectrum of 2MASS J1457-3543 (blue) with the available photometric data (red dots) from the literature for checking the flux calibration, and the BT-Settl model (black) for double-checking the estimated $T_{\text {eff }}$ and $\log g$.

target (indicated as a downward arrow in Fig. 8b). Its estimated $T_{\text {eff }}$ and $\log g$ are confirmed by fitting a BT-Settl model with the same qualities to its spectrum (Fig. 5).

Another possibility is that the LiI line is absent in the spectrum of this target. In this case, it is possible that the object is older than the UCL sub-association. By comparing the models of Baraffe et al. (2015) and empirical results of $\mathrm{Li}$ distribution in the $\sim 50$ Myr-old IC 2391 open cluster (Barrado y Navascués et al. 2001), we reach the conclusion that Li nondetection implies 2MASS J1457-3543 is older than 80 Myr.

An age of 80-150 Myr (e.g., similar to the Pleiades) can still be compatible with a high activity level and fast rotation. Alternatively, activity and rotation might be induced by a close companion through tidal locking. Some indications of an unresolved binary are suggested by the astrometric excess noise reported for this object, which is equal to 0.99 mas with a significant astrometric-excess-noise sigma of 3.29. However, a very close, tidally locked binary is not expected to cause significant astrometric effects. In any case, while the spectral lines are very broad, there are no indications of blending of additional components. Therefore, the possibility of a binary object remains speculative at this stage.

We also put 2MASS J1457-3543 on a CMD with other UCL members (Damiani et al. 2019), plus our other target 2MASS J1517-3028 located in the same sub-association (Fig. 6). This CMD re-confirms 2MASS J1517-3028 as a PMS member of UCL, and also shows that 2MASS J1457-3543 is fainter than the UCL members studied by Damiani et al. (2019). We calculated RUWE of the M-type objects in this sample and found that 68 out of 588 of them have RUWE $>1.4$ (Fig. 6). The SpTs of the members are calculated according to their $M_{\mathrm{G}}$ (Kiman et al. 2019). The RUWE of 2MASS J1457-3543, although intrinsically fainter than the members, is equal to 1.174 that is below RUWE $\sim 1.4$, further decreasing the chance of our target being a nonsingle object.

We also checked whether the large $v \sin i$ of 2MASS J14573543 is compatible with its SpT. According to Jeffers et al. (2018), there is a correlation between the $\mathrm{H}_{\alpha}$ activity and $v \sin i$ of M-type dwarfs: the trend shows earlier SpTs have lower rotation and activity levels, while later SpTs are mostly fast rotating and chromospherically active stars. The studied sample in Jeffers et al. (2018) is not sufficiently large to verify such a correlation, but it seems 2MASS J1457-3543 falls in the latter 


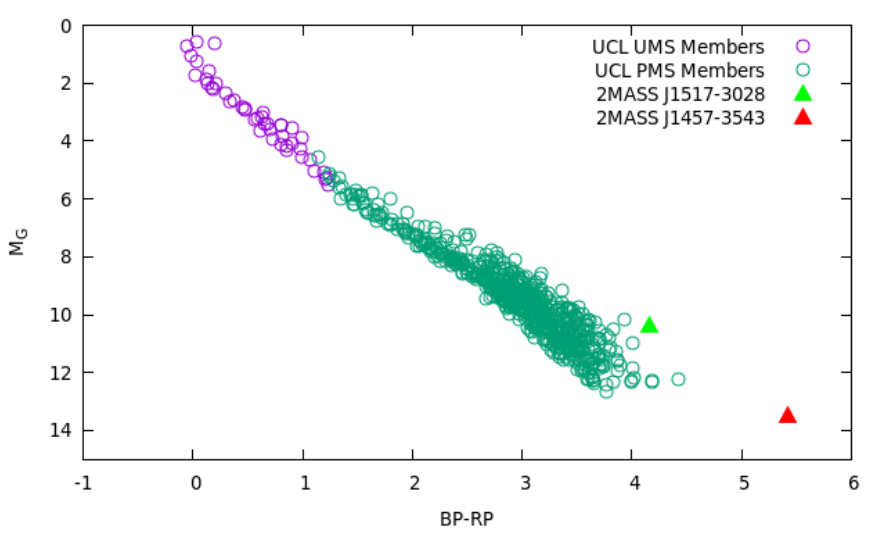

(a)

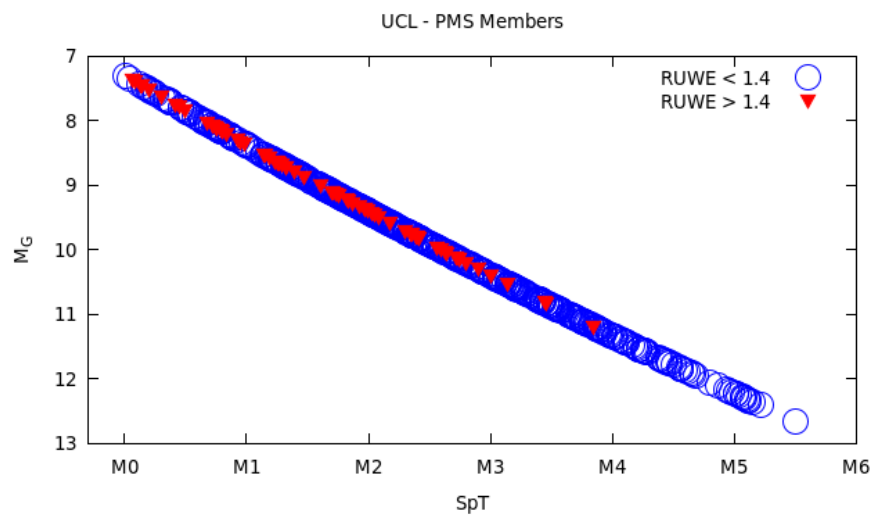

(b)

Fig. 6. Panel a: UMS and PMS members of UCL (Damiani et al. 2019) with our targets located in the same sub-association, namely, 2MASS J1457-3543 and 2MASS J1517-3028. Panel b: RUWE of M-type members of UCL. The blue circles indicate the members with RUWE indices below the threshold of 1.4, while the red triangles represent members with RUWE $>1.4$. Only 68 of 588 objects have a significant RUWE.

category of late M-type dwarfs which are fast rotating and active. However, the reported $v \sin i=62.0 \mathrm{~km} \mathrm{~s}^{-1}$ is higher than the values found in the Carmencita catalog for the same SpT in the same paper. We note that higher values were obtained for earlier and later M-type dwarfs in the same catalog. A larger sample was studied by Konopacky et al. (2012), who focused on determining the rotational velocities of very late-type, low-mass individual binary components. According to this latter work, rotational velocities as high as the value found for 2MASS J1457-3543 (of M8 SpT or later) had been identified before in the literature. More importantly, Dahm et al. (2012) studied 94 members of Upper Scorpius (USC) two of which have an M8 SpT, with measured projected rotational velocities of $22.70 \pm 4.47$ and $60.94 \pm 3.03 \mathrm{~km} \mathrm{~s}^{-1}$. The latter, which has a similar rotational velocity to our target, is identified as a fast rotator. In total, 4 out of 38 objects with SpTs between M4 and M8 possess rotational velocities higher than $30 \mathrm{~km} \mathrm{~s}^{-1}$, which confirms that the rotational velocity of our target is not uncommon among very young objects.

From $v \sin i$ and stellar radius, we estimated a rotation period of 0.2 days for 2MASS J1457-3543. Recent studies on $\mathrm{M}$ dwarfs belonging to Pleiades and Praesepe clusters (Somers et al. 2017) and low-mass stars residing in USC and $\rho$ Ophiuchus (Rebull et al. 2018) suggest that rotation periods
Table 6. Photometry of our targets.

\begin{tabular}{lccccc}
\hline \hline Name & $\begin{array}{c}B P-R P \\
(\mathrm{mag})\end{array}$ & $\begin{array}{c}J \\
(\mathrm{mag})\end{array}$ & $\begin{array}{c}H \\
(\mathrm{mag})\end{array}$ & $\begin{array}{c}K s \\
(\mathrm{mag})\end{array}$ & $\begin{array}{c}G \\
(\mathrm{mag})\end{array}$ \\
\hline 2MASS J1815-3249 & 2.25 & 11.07 & 10.44 & 10.2 & 13.6 \\
2MASS J1517-3028 & 3.18 & 12.54 & 11.93 & 11.64 & 15.8 \\
2MASS J1324-5129 & 3.87 & 14.59 & 14.04 & 13.63 & 18.71 \\
2MASS J1457-3543 & - & 14.13 & 13.5 & 13.1 & 18.52 \\
2MASS J1549-3539 & 2.75 & 14.85 & 14.08 & 13.82 & 18.37 \\
\hline
\end{tabular}

much shorter than 1 day are frequent for the lowest mass objects $\left(\leq 0.1 M_{\odot}\right)$. Finally, the position of 2MASS J1457-3543 on a CMD is well above the main sequence (Fig. 8a) and along the sequence of Sco-Cen members. However, it is also possible that the overluminosity is due to binarity.

We conclude that the most likely scenario explaining the spectrum of 2MASS J1457-3543 is that the target is a young (17.75 $\pm 4.15 \mathrm{Myr}$ ) and active late-type $\mathrm{M}$ dwarf and a new member of the UCL. Nevertheless, both of the other alternatives for the target, namely (i) a highly active, fast-rotating young object with an approximate age of $100 \mathrm{Myr}$, or (ii) a close binary, remain viable. As we cannot exclude that the observed spectrum is that of a spectroscopic binary, the stellar parameters we derive from it must be taken with caution, as they are only reliable if the object is a single star. However, it is noteworthy that if our evaluations of 2MASS J1457-3543 as a single object are accurate, this target might be a very wide companion of HIP 73145. The RV $=4.01 \pm 8.87 \mathrm{~km} \mathrm{~s}^{-1}$ and age $(17.75 \pm 4.15 \mathrm{Myr})$ of 2MASS J1457-3543 are consistent with those of HIP 73145 ( RV $=3.8 \pm 1.6 \mathrm{~km} \mathrm{~s}^{-1}$, age $\sim 15 \mathrm{Myr}$ ), although their inconsistent parallax and the wide projected separation argues against their physical association.

Finally, as the age of this object is highly uncertain, its mass cannot be unambiguously estimated. We point out that 2MASS J1457-3543 should be substellar $\left(<0.069 M_{\odot}\right)$ if the object is younger than $144.5 \mathrm{Myr}$. Masses of 0.03 and $0.06 M_{\odot}$ are derived for 15.5 Myr (Sco-Cen age) and 120 Myr (Pleiades age), respectively.

\section{Discussion and conclusions}

We analyzed the X-shooter spectra of probable wide companions of four different stellar systems to determine their physical parameters. 2MASS J1815-3249 was found to be a field object unrelated to the $\beta$-Pictoris $\mathrm{MG}$, with an estimated age of $>150 \mathrm{Myr}$, in spite of its parallax and proper motions, which are similar to the bona-fide member V4046 Sgr. The results we obtained for the other three targets located in Sco-Cen are summarized below. All the four wide companion candidates analyzed in this paper are placed on a CMD together with the objects studied in Pecaut \& Mamajek (2016) (featured as blue dots in Fig. 8a). This CMD confirms 2MASS J1324-5129 and 2MASS J1517-3028 as PMS members of Sco-Cen sub-associations. For 2MASS J1457-3543, considering all the aspects addressed in the previous section, we find that the most likely scenario indicated by the target spectrum is that of a single object and a PMS (evident from Fig. 8a) member of UCL. Following this scenario, 2MASS J1457-3543 could also be an ultra-wide companion to HIP 73145 considering their consistent ages and RVs, although their discrepant parallax reported by Gaia DR2 argues against this possibility. 
Table 7. Equivalent width of the relevant lines indicating chromospheric and accretion tracers for our candidates.

\begin{tabular}{lcccc}
\hline \hline Name & $\begin{array}{c}E W_{\mathrm{LiI}} \\
(\mathrm{nm})\end{array}$ & $\begin{array}{c}E W_{\mathrm{H}_{\alpha}} \\
(\mathrm{nm})\end{array}$ & $\begin{array}{c}E W_{\mathrm{H}_{\beta}} \\
(\mathrm{nm})\end{array}$ & $\begin{array}{c}E W_{\mathrm{H}_{\gamma}} \\
(\mathrm{nm})\end{array}$ \\
\hline 2MASS J1815-3249 & $<0.0036^{(a)}$ & $0.012 \pm 0.001$ & $-0.034 \pm 0.003$ & $-0.013 \pm 0.005$ \\
2MASS J1517-3028 & $0.038 \pm 0.008$ & $-1.083 \pm 0.055$ & $-1.019 \pm 0.067$ & $-1.126 \pm 0.201$ \\
2MASS J1324-5129 & $0.070 \pm 0.020$ & $-1.068 \pm 0.142$ & $-0.659 \pm 0.149$ & $-0.723 \pm 0.129$ \\
2MASS J1457-3543 & $<0.039^{(a)}$ & $-0.983 \pm 0.090$ & $-0.789 \pm 0.166$ & $-0.886 \pm 0.135$ \\
2MASS J1549-3539 & $0.035 \pm 0.010$ & $-9.943 \pm 0.252$ & $-4.085 \pm 0.701$ & $-2.722 \pm 0.217$ \\
\hline
\end{tabular}

Notes. ${ }^{(a)}$ Three-sigma upper limits (see the text for further explanation). The $E W_{\mathrm{LiI}}$ of 2MASS J1549-3539 may be affected by veiling (Alcalá et al. 2020).

Table 8. Wide-companion membership status checklist for our targets.

\begin{tabular}{|c|c|c|c|c|c|}
\hline Name & $\begin{array}{c}\text { Consistent kinematic properties } \\
\text { with the stellar system } \\
\text { (yes/no) }\end{array}$ & $\begin{array}{c}\text { Age } \\
(\mathrm{MS} / \mathrm{PMS})\end{array}$ & $\begin{array}{l}\text { Active } \\
\text { (yes/no) }\end{array}$ & $\begin{array}{l}\text { Contains Li I } \\
\text { (yes/no) }\end{array}$ & Conclusion \\
\hline 2MASS J1815-3249 & no & MS & no & no & Field object \\
\hline 2MASS J1517-3028 & yes & PMS & yes & yes & $\begin{array}{c}\text { UCL member }+ \\
\text { HIP } 74865 \text { probable wide companion }\end{array}$ \\
\hline 2MASS J1324-5129 & no & PMS & yes & yes & LCC new member \\
\hline 2MASS J1457-3543 & no & PMS & yes & Ambiguous & Ambiguous \\
\hline 2MASS J1549-3539 & yes & PMS & yes & yes & $\begin{array}{l}\text { Lupus I new member }+ \\
\text { GQ Lup probable wide companion }\end{array}$ \\
\hline
\end{tabular}

The criteria used for distinguishing between accreting and nonaccreting objects are those proposed by White \& Basri (2003), which are based on the EW of $\mathrm{H}_{\alpha}$ as a function of SpT and the full width of the line profile at $10 \%$ of the peak. The plot of $E W_{\mathrm{H}_{\alpha}}$ against SpT (Fig. 7) for our candidates and the T Tauri stars studied by Manara et al. (2013) suggests that the only accreting object in our sample is 2MASS J1549-3539, which was fully investigated by Alcalá et al. (2020). A checklist of the properties of our targets relevant for their wide-companion membership status is presented in Table 8 .

$E W_{\mathrm{LiI}}$ of $\beta$-Pictoris MG and Lupus members measured by Messina et al. (2016) and Biazzo et al. (2017), respectively, are plotted along with the $E W_{\mathrm{Li} \text { I }}$ of our targets; see Fig. $8 \mathrm{~b}^{7}$. Our only target located in $\beta$-Pictoris MG, namely 2MASS J18153249 , which is determined as a field object, is depleted of lithium as expected, taking into account its age (older than $150 \mathrm{Myr}$ ). Members of $\beta$-Pictoris MG depleted of lithium pinpoint the age of the association, as lithium is burned rapidly in low-mass stars due to PMS contraction increasing stellar core temperature to as high as $\sim 3 \times 10^{6} \mathrm{~K}$. Subsequently, depending on the mass of the stars, at a certain age, convective mixing depletes the stars of lithium entirely. The lithium gap existing among $\beta$-Pictoris MG members (blue dots) indicates the older age of these cluster with respect to Lupus members (purple dots). Our three remaining candidates, which we acknowledge as Lupus members, have consistent $E W_{\mathrm{Li} \text { I }}$ with members of the association. Taking into consideration the ambiguities involved in analyzing the spectrum of 2MASS J1457-3543 (see Sect. 4.4), we only report a threesigma upper limit for $E W_{\mathrm{Li} \text { I }}$ of this object.

\footnotetext{
7 The $V$ band magnitude for our program targets was derived from the Pecaut \& Mamajek (2013) tables (the updated table can be found online at http://www . pas.rochester. edu/ emamajek/EEM_dwarf_ UBVIJHK_colors_Teff.txt
}

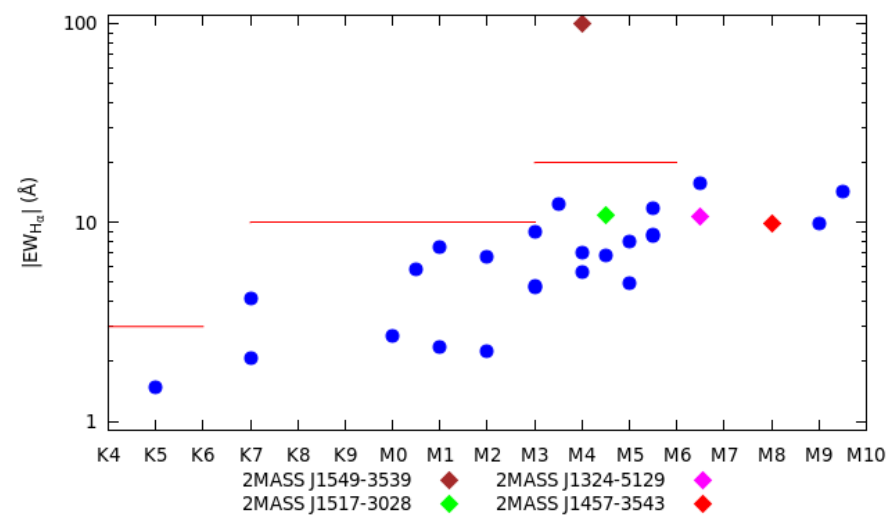

Fig. 7. $E W_{\mathrm{H}_{\alpha}}$ vs. $\mathrm{SpT}$ of our targets with $\mathrm{H}_{\alpha}$ emission (diamonds) along with the objects studied by Manara et al. (2013, blue dots). The red horizontal lines represent the thresholds between nonaccreting and accreting objects for different SpT ranges according to White \& Basri (2003).

As a final test, we also measured the total velocity difference $(\Delta v)$ between our targets and their corresponding central stars. This quantity can be compared with $\Delta v_{\max }(s)$, that is, the maximum total velocity difference as a function of projected separation between the two binary components in astronomical units as suggested by Andrews et al. (2017) for binaries of a total mass of $10 M_{\odot}$. This comparison provides clues as to whether the possible wide companions studied in this work are truly gravitationally bound systems. As expected, we obtained large error bars associated with $\Delta v$ of 2MASS J1517-3028 and 2MASS J1549-3539 (Fig. 9). The resultant values considering their large error bars are consistent with $\Delta v_{\max }$ (orange line in Fig. 9), but drawing any firm conclusions regarding the physical association of the two objects is not possible because of these large error bars. This is partly due to large errors associated with 


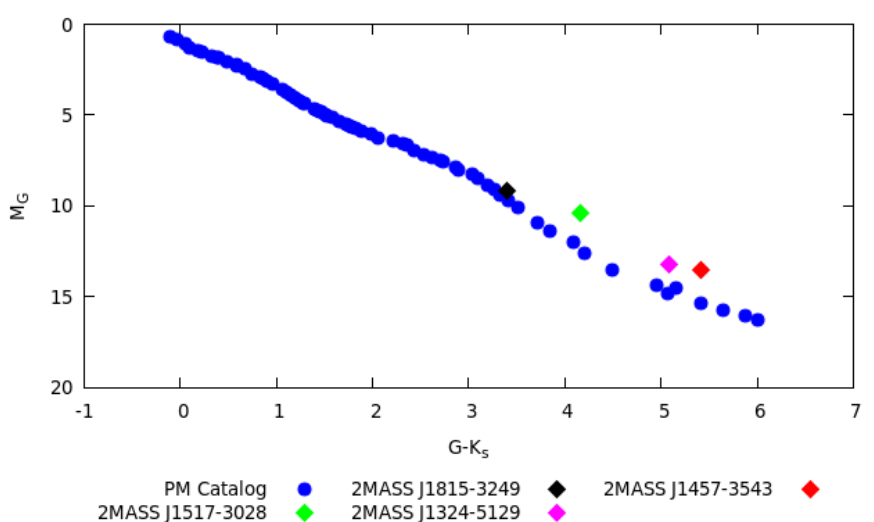

(a)

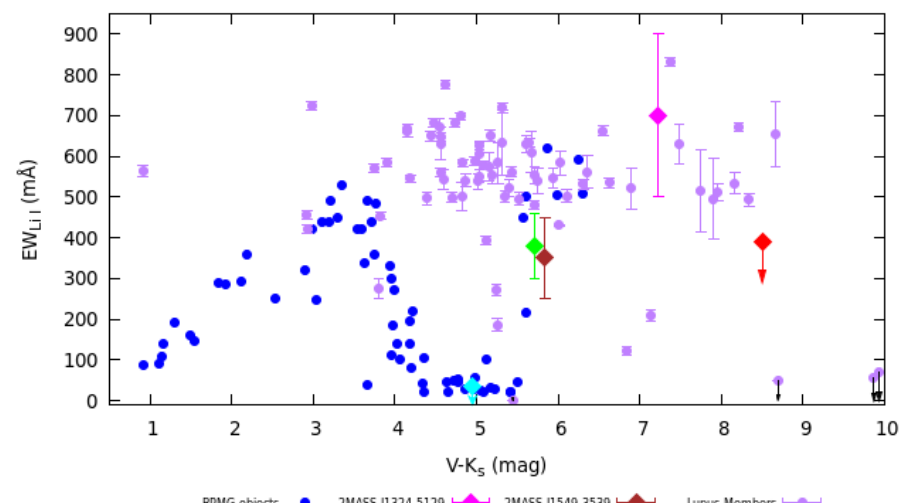

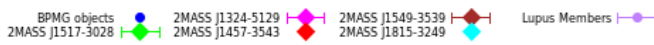

(b)

Fig. 8. Panel $a$ : CMD of the wide companion candidates in this work (diamond) together with the objects in Pecaut \& Mamajek (2013, blue dots). Panel $b: E W_{\mathrm{Li}}$ of our targets (diamonds) plotted with Lupus members (Biazzo et al. 2017) and 66 members of the $\beta$ Pictoris association (Messina et al. 2016).

the RV found for these objects and the multiplicity of the stellar systems. Thus, we still cannot conclude whether or not these two targets are gravitationally bound to their associated central stars. For 2MASS J1324-5129, on the other hand, we can rule out the possibility that this target is bound to HIP 65426 as its $\Delta v$ significantly exceeds its $\Delta v_{\max }$. As discussed by Ramírez et al. (2019), this statement is true if there is no other effect influencing the kinematic properties of the objects involved, such as an unseen companion to any of the components or unknown systematics in Gaia DR2.

2MASS J1517-3028. According to our analysis, this target is a highly probable wide companion of HIP 74865 with an estimated age of 7-8 Myr -younger than the UCL subassociation $(15 \pm 3 \mathrm{Myr})$ in which the object is located. This age discrepancy has been addressed previously (Feiden 2016; Asensio-Torres et al. 2019) as theoretical isochrones failing to reproduce the correct age of $\mathrm{K}$ and $\mathrm{M}$ stars by a factor of two (due to neglecting magnetic fields in the models). However, this issue has only been flagged up for 2MASS J1517-3028, and not for 2MASS J1324-5129 or 2MASS J1457-3543, although these latter two are also late-type M-dwarfs, albeit later in type in comparison with 2MASS J1517-3028. According to Baraffe et al. (2015) models, the estimated ages for 2MASS J1324-5129 and 2MASS J1457-3543 meet our expectations and are highly

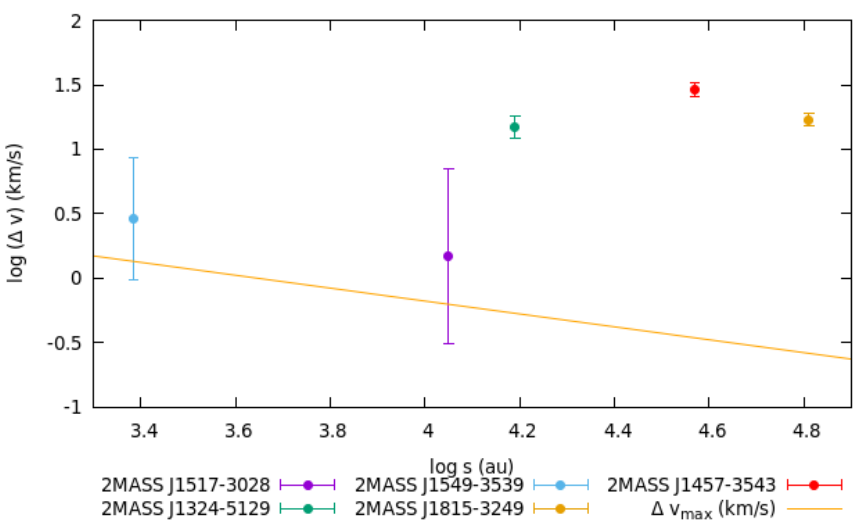

Fig. 9. $\log -\log$ plot of total velocity difference $\Delta v\left(\mathrm{~km} \mathrm{~s}^{-1}\right)$ vs. projected separation $s(\mathrm{au})$ for the five plausible wide companions studied in this work. $\Delta v_{\max }\left(\mathrm{km} \mathrm{s}^{-1}\right)$ (orange line) indicates the maximum total velocity difference that bound binaries with a total mass equal to $10 M_{\odot}$ in circular orbits can possess; see Ramírez et al. (2019) for details.

consistent with the age of the stellar sub-associations they are located in. We noticed that, based on the EW of Balmer emission lines and infrared ca II triplet lines, 2MASS J1517-3028 is magnetically more active than 2MASS J1324-5129 and 2MASS J1457-3543. Therefore, it might be true that the acquired age for 2MASS J1517-3028 based on Baraffe et al. (2015) models (that neglect the effect of magnetic fields) is younger than its real value by a factor of two. Assuming the whole stellar system as a triple system, HIP 74865 hosts a brown dwarf companion at a small separation with an M-dwarf star at a larger separation. As an already confirmed PMS member of UCL, our age estimation and determined stellar parameters reconfirm the membership of this target. According to BANYAN $\Sigma$ (Gagné et al. 2018), with the RV calculated for this object in the present paper, its membership probability is higher than $98 \%$.

2MASS J1324-5129. Despite its similar kinematic properties to HIP 65426, their corresponding total velocity difference rules out the possibility that 2MASS J1324-5129 is bound to this stellar system. Therefore, we conclude that 2MASS J13245129 is a typical young $(16 \pm 2.2 \mathrm{Myr})$ and active M-dwarf and a highly probable - approximately $94 \%$ according to BANYAN $\Sigma$ (Gagné et al. 2018) - member of LCC (16 \pm 2 Myr) based on its kinematic properties and estimated age.

2MASS J1457-3543. We were not able to decipher whether the spectrum belongs to a single object or an unresolved binary, and therefore cannot form any firm conclusions regarding the membership and acquired physical parameters of this object.

2MASS J1549-3539. This target is a highly probable wide companion of GQ Lupi, possibly making the stellar system a triple system consisting of a brown dwarf at a close separation (GQ Lupi b) and an M-dwarf at a larger distance. GQ Lupi b is a strong accretor with a surrounding disk, similarly to 2MASS J1549-3539. According to BANYAN $\Sigma$, the probability of 2MASS J1549-3539 being a member of UCL in the Sco-Cen association is above $97 \%$.

Considering the findings of the present paper, we therefore update the status of the central stars of these systems as follows:

V4046 Sgr. As its wide companion candidate, 2MASS J1815-3249, turned out to be an unrelated field object, this stellar system remains a triple system, consisting of a very close binary with similar components (V4046 Sgr itself) and a wide companion (GSC 7396-00759). The different evolutionary phases of 
the disks surrounding the two objects are highly interesting and merit further investigation.

HIP 74865 and GQ Lupi. These two systems have similar architectures, with a BD orbiting the most massive star in the system, plus a low-mass star at a very wide separation. Besides the dynamical environment, the additional component (especially for the early type primary HIP 74865) might allow us to refine the age of the system. It is noteworthy that GQ Lup C is different in this regard: because of its strong absorption lines and edge-on disk, it is not easy to use the information offered by this target for determining the age of the system. However, in both cases there is the caveat that we are not sure if the proposed wide companions are gravitationally bound to their associated central stars, although close separations and similar kinematic properties strongly favor a common origin.

HIP 65426. We rule out the physical association of this star with 2MASS J1324-5129. Hence, the sole companion of HIP 65426 is the planet it hosts. Marleau et al. (2019) modeled the formation of HIP $65426 \mathrm{~b}$ in the core-accretion framework and identified the most probable path as an outward scattering after a phase of dynamical instability. The lack of wide stellar companions from Gaia supports an origin internal to the planetary system based on this (hypothetical) framework, although a triggering by a passing star within the native association cannot be ruled out.

HIP 73145. This star is single as we ruled out its physical association with our candidate wide companion 2MASS J14573543. We were hoping that finding a companion gravitationally bound to this star could provide us with hints on the formation of the multi-belt architecture of its disk. The lack of stellar and massive brown dwarf companions over the full range of separations suggests that these features in the disk are not linked to external objects.

The two triple systems presented in this paper have a similar configuration, both hosting a sub-stellar object in a close orbit around the central star and a low-mass stellar companion at a larger distance. Regarding GQ Lupi, the central and companion stars are all surrounded by disks. On the contrary, for HIP 74865, no IR excess or signs of the presence of a disk around the central or companion stars are detected. As opposed to these configurations, there are other triple system architectures such as HD $284149 \mathrm{ABb}$ (Bonavita et al. 2017) in which the substellar companion (HD 284149 b) has a larger separation from the central star compared to the low-mass stellar companion (HD 284149 B). There is no hint of a circumstellar disk around the central star. Various triple-system configurations of this type suggest different formation mechanisms are at play. As few such triple systems have been discovered, we cannot draw robust conclusions regarding the different formation mechanisms able to produce such configurations. However, it might be interesting to explore the currently identified stellar systems at larger distances to find out whether they possess PMS ultra-wide companions, hinting at larger multiple systems. Such studies would be valuable because multiple systems are three times more likely to have a distant companion within $10 \mathrm{kau}$ compared to single stars. On the other hand, ultra-wide companions are biased towards high multiplicity at shorter separations (Joncour et al. 2017). If genuine, it is plausible that the aforementioned discovered systems also follow a cascade fragmentation scenario of the natal molecular core as suggested by Joncour et al. (2017).
Acknowledgements. We are grateful to Dr. Julio Chanamé, the referee of this paper, whose comments significantly improved this work. We warmly thank G. Catanzaro for helping us in deriving the RV of HIP 73145, and S. Petrus for sharing his results on the HIP 65426 system before the publication. We thank C. F. Manara for his help during the preparation phase of the X-shooter observations and for his useful comments and suggestions. This work has made use of data from the European Space Agency (ESA) mission Gaia (https://www. cosmos.esa.int/gaia), processed by the Gaia Data Processing and Analysis Consortium (DPAC, https://www. cosmos.esa.int/web/gaia/dpac/ consortium). Funding for the DPAC has been provided by national institutions, in particular the institutions participating in the Gaia Multilateral Agreement. This research has made use of the SIMBAD database and Vizier services, operated at CDS, Strasbourg, France. This research has made use of the services of the ESO Science Archive Facility. We acknowledge financial support from the ASI-INAF agreement n.2018-16-HH.0. JMA acknowledges financial support from INAF thourgh the project PRIN-INAF-MAIN-STREAM 2017 "Protoplanetary disks seen through the eyes of new-generation instruments".

\section{References}

Alcalá, J. M., Manara, C., Natta, A., et al. 2017, A\&A, 600, A20 Alcalá, J. M., Majidi, F. Z., Desidera, S., et al. 2020, A\&A, 635, L1 Alden, H. L. 1928, AJ, 39, 20

Allard, F., Homeier, D., \& Freytag, B. 2012, RSPTA, 370, 2765

Alonso-Floriano, F. J., Caballero, J. A., Cortés-Contreras, M., et al. 2015, A\&A, 583, A85

Andrews, J. J., Chanamé, J., Agueros, M. A., et al. 2017, MNRAS, 472, 675 Asensio-Torres, R., Currie, T., Janson, M., et al. 2019, A\&A, 622, A42 Barrado y Navascués, D., Deliyannis, C. P., \& Stauffer, J. R. 2001, ApJ, 549, 452 Baraffe, I., Homeier, D., Allard, F., \& Chabrier, G. 2015, A\&A, 577, A42

Beuzit, J. L., Vigan, A., Mouillet, D., et al. 2019, A\&A, 631, A155 Biazzo, K., Frasca, A., Alcalá, J. M., et al. 2017, A\&A, 605, A66 Bonavita, M., D’Orazi, V., Mesa, D., et al. 2017, A\&A, 608, A106 Caballero, J. A. 2009, A\&A, 507, 251

Cayrel, R. 1988, Proceedings of the Alpbach Summer school

Chanamé, J., \& Gould, A. 2004, ApJ, 601, 289

Chanamé, J., \& Ramírez, I. 2012, ApJ, 746, 102

Chauvin, G., Desidera, S., Lagrange, A. M., et al. 2017, A\&A, 605, L9

Chen, C. H., Mamajek, E. E., Bitner, M. A., et al. 2011, ApJ, 738, 122

Choi, J., Dotter, A., Conroy, C., et al. 2016, ApJ, 823, 102

Connelley, M. S., Reipurth, B., \& Tokunaga, A. T. 2008, AJ, 135, 2526

Dahm, S. E., Slesnick, C. L., \& White, R. J. 2012, ApJ, 745, 56

Damiani, F., Prisinzano, L., Pillitteri, I., et al. 2019, A\&A, 623, A112

Deacon, N. R., \& Kraus, A. L. 2020, MNRAS, 496, 5176

Desidera, S., Chauvin, G., Bonavita, M., et al. 2020, A\&A, submitted

D’Orazi, V., Gratton, R., Desidera, S., et al. 2019, Nat. Astron., 3, 167

Dotter, A. 2016, ApJS, 222, 8

Duchéne, G., Delgado-Donate, E., Haisch, K. E., Loinard, L., \& Rodriguez, L.

F. 2007, in Protostars and Planets V, ed. B. Reipurth, D. Jewitt, \& K. Keil (Tucson, AZ: Univ. Arizona Press), 379

El-Badry, K., \& Rix, H. W. 2019, MNRAS, 482, L139

Feiden, G. A. 2016, A\&A, 593, A99

Feldt, M., Olofsson, J., Boccaletti, A., et al. 2017, A\&A, 601, A7

Feng, F., Anglada-Escudé, G., Tuomi, M., et al. 2019, MNRAS, 490, 5002

Frasca, A., Biazzo, K., Lanzafame, A. C., et al. 2015, A\&A, 575, A4

Frasca, A., Biazzo, K., Alcalá, J. M., et al. 2017, A\&A, 602, A33

Freudling, W., Romaniello, M., Bramich, D. M., et al. 2013, A\&A, 559, A96

Gaia Collaboration (Brown, A. G. A., et al.) 2018, A\&A, 616, A1

Garcés, A., Catalán, S., \& Ribas, I. 2011, A\&A, 531, A7

Garufi, A., Benisty, M., Stolker, T., et al. 2017, ESO Messenger, 169, 32

Gagné, J., Mamajek, E. E., Malo, L., et al. 2018, ApJ, 856, 23

Ghez, A. M., Neugebauer, G., \& Matthews, K. 1993, AJ, 106, 2005

Hinkley, S., Kraus, A. L., Ireland, M. J., et al. 2015, ApJ, 806, L9

Innes, R. T. A. 1915, Circular of the Union Observatory Johannesburg, 30, 235

Innes, R. T. A. 1926, Circular of the Union Observatory Johannesburg, 70, 390

Jeffers, S. V., Schöfer, P., Lamert, A., et al. 2018, A\&A, 614, A76

Jiang, Y. F., \& Tremaine, S. 2010, MNRAS, 401, 977

Joncour, I., Duchłne, G., \& Moraux, E. 2017, A\&A, 599, A14

Kaib, N. A., Raymond, S. N., Duncan, M., et al. 2013, Nature, 493, 381

King, R. R., McCaughrean, M. J., Homeier, D., et al. 2010, A\&A, 510, A99

Keppler, M., Benisty, M., Müller, A., et al. 2018, A\&A, 617, A44

Kervella, P., Thévenin, F., \& Lovis, C. 2017, A\&A, 598, L7

Kiman, R., Schmidt, S. J., Angus, R., et al. 2019, AJ, 157, 231

Konopacky, Q. M., Ghez, A. M., Fabrycky, D. C., et al. 2012, ApJ, 750, 1

Kouwenhoven, M. B. N., Goodwin, S. P., \& Parker, R. J. 2010, MNRAS, 404 1835

Kraus, A. L., \& Hillenbrand, L. A. 2007, AJ, 134, 2340 
Lazzoni, C., Gratton, R., Alcalá, J., et al. 2020, A\&A, 635, L11

Lindegren, L., Lammers, U., Hobbs, D., et al. 2012, A\&A, 538, A78

Lindegren, L., Hernández, J., Bombrun, A., et al. 2018, A\&A, 616, A2

López-Santiago, J., Montes, D., Galvez-Ortiz, M. C., et al. 2010, A\&A, 514, A97

Luyten, W. J. 1925, Harvard College Observatory Bulletin, 818, 2

MacGregor, M. A., Wilner, D. J., Czekala, I., et al. 2017, ApJ, 835, 17

Madsen, S., Dravins, D., \& Lindegren, L. 2002, A\&A, 381, 446

Manara, C. F., Testi, L., Rigliaco, E., et al. 2013, A\&A, 551, A107

Manara, C. F., Frasca, A., Alcalá, J. M., et al. 2017, A\&A, 605, A86

Marleau, G. D., Coleman, G. A. L., Leleu, A., \& Mordasini, C. 2019, A\&A, 624, A20

Martinez-Arnáiz, R., López-Santiago, J., Crespo-Chacón, I., et al. 2011, MNRAS, 414, 2629

Messina, S., Lanzafame, A. C., Feiden, G. A., et al. 2016, A\&A, 596, A29

Modigliani, A., Goldoni, P., \& Royer, F. 2011, Proc. SPIE, 7737, 773728

Moeckel, N., \& Clarke, C. J. 2011, MNRAS, 415, 1179

Neuhäuser, R., Mugrauer, M., Seifahrt, A., et al. 2008, A\&A, 484, 281

Paxton, B., Marchant, P., Schwab, J., et al. 2015, ApJS, 220, 15

Pecaut, M. J., \& Mamajek, E. E. 2013, ApJS, 208, 9

Pecaut, M. J., \& Mamajek, E. E. 2016, MNRAS, 461, 794

Pecaut, M. J., Mamajek, E. E., \& Bubar, E. J. 2012, ApJ, 746, 154

Petrus, S., Bonnefoy, M., Chauvin, G., et al. 2020, A\&A, 633, A124

Quast, G. R., Torres, C. A. O., de la Reza, R., et al. 2000, IAU Symp., 200

Quinn, D. P., Wilkinson, M. I., Irwin, M. J., et al. 2009, MNRAS, 396, L11
Ramírez, I., Khanal, S., Lichon, S. J., et al. 2019, MNRAS, 490, 2448

Rebull, L., Stauffer, J., Cody, A. M., et al. 2018, AJ, 155, 196

Reylé, C. 2018, A\&A, 619, L8

Riddick, F., Roche, P., \& Lucas, P. 2007, MNRAS, 381, 1067

Rizzuto, A. C., Ireland, M. J., \& Robertson, J. G. 2011, MNRAS, 416, 3108

Rosen, S. R., Webb, N. A., Watson, M. G., et al. 2016, A\&A, 590, A1

Rosenfeld, K. A., Andrews, S. M., Wilner, D. J., et al. 2013, ApJ, 775, 136

Scholz, R. D., McCaughrean, M. J., Lodieu, N., \& Kuhlbrodt, B. 2003, A\&A 389, L29

Schwarz, H., Ginski, C., de Kok, R. J., et al. 2016, A\&A, 593, A74

Sissa, E., Olofsson, J., Vigan, A., et al. 2018, A\&A, 613, L6

Somers, G., Stauffer, J., Rebull, L., Cody, A. M., \& Pinsonneault, M. 2017, ApJ, 850,134

Song, I., Zuckerman, B., \& Bessel, M. S. 2008, ApJ, 599, 342

Stempels, H. C., \& Gahm, G. F. 2004, A\&A, 421, 1159

Tian, H., Liu, C., Xu, Y., et al. 2019, ApJ, 871, 184

Tobin, J. J., Looney, L. W., Li, Z.-Y., et al. 2016, ApJ, 818, 73

Torres, C. A. O., Quast, G. R., Melo, C. H. F., \& Sterzik, M. F. 2008, in Featured

in Handbook of Star Forming Regions, ed. Bo Reipurth, The Southern Sky, 2, 757

Vernet, J., Dekker, H., D’Odorico, S., et al. 2011, A\&A, 536, A105

Wang, Y., Zhou, J., Hui-gen, L., et al. 2017, ApJ, 848, 20

Weinberg, M. D., Shapiro, S. L., \& Wasserman, I. 1987, ApJ, 31

White, R. J., \& Basri, G. 2003, ApJ, 582, 1109

Zhou, Y., Herczeg, G. J., Kraus, A. L., et al. 2014, ApJ, 783, L17 